Pacific

Journal of

Mathematics

FINITE RELATIVE DETERMINATION AND RELATIVE STABILITY

León Kushner and Brasil Terra Leme

Volume $192 \quad$ No. 2

February 2000 


\title{
FINITE RELATIVE DETERMINATION AND RELATIVE STABILITY
}

\author{
León Kushner and Brasil Terra Leme
}

\begin{abstract}
This paper is divided in three parts. The first part deals with the equivalence of finite determination on the right and finite relative determination (with respect to $S$ ) under some conditions on $S$. The second part deals with infinite determinacy (with respect to $S$, a germ of a closed set of $\mathbb{R}^{n}$ ). Both generalize results of $\mathrm{P}$. Porto $[\mathrm{P}]$ for a big family of closed subsets $S$ of $\mathbb{R}^{n}$. The third part is a special case which is quite interesting, when $S$ coincides with the closure of its interior.
\end{abstract}

\section{Introduction.}

This paper continues the work done in $[\mathbf{K}]$. In that paper there were proven results of finite relative determination for particular algebraic subsets of $\mathbb{R}^{n}$. Here we continue in this direction. In the first part we prove the equivalence of finite determination on the right and finite relative determination for a big family of algebraic subsets, generalizing the results of $[\mathbf{P}-\mathbf{L}]$. In the second part we continue with the concept of infinite determinacy and remarking the importance of quasihomogeneous polynomials. In the third part we generalize the results on relative stability in $[\mathbf{P}-\mathbf{L}]$ and $[\mathbf{P}]$ for a broader family of closed subsets of $\mathbb{R}^{n}$, such as good semianalytic subsets.

\section{Notation.}

We shall work in $\mathcal{E}(n)$, the local algebra of $C^{\infty}$ function germs of $\mathbb{R}^{n}$ to $\mathbb{R}$ around the origin with maximal ideal $m(n)$. The powers of $m(n)$ will be denoted by $m(n)^{k}$ and $m(n)^{\infty}=\cap_{k=1}^{\infty} m(n)^{k}$. For $I=\left(i_{1}, \ldots, i_{n}\right)$ a multiindex of natural numbers and $x=\left(x_{1}, \ldots, x_{n}\right)$ we shall write $x^{I}=x_{1}^{i_{1}} \ldots x_{n}^{i_{n}}$ and $|I|=i_{1}+\cdots+i_{n}$, also for a germ $f, \frac{\partial^{|I|} f}{\partial x^{I}}=\frac{\partial^{|I|} f}{\partial x_{1}^{i_{1}} \ldots \partial x_{n}^{i_{n}}}$.

For $S$ a subset of $\mathbb{R}^{n}, \overline{0} \in S, \operatorname{cl}(S)$ and $\operatorname{int}(S)$ will denote the closure and the interior of $S$ respectively and $G_{S}$ will be the group of germs of diffeomophisms $\phi$ at $\overline{0}$, such that $\phi(x)=x \forall x \in S$. Also $d(x, S)$ will denote the usual distance from the point $x$ to the subset $S$.

Finally if $f$ is a germ, $\partial f / \partial x_{i}$ will be the partial derivatives of $f$ and $\left\langle\frac{\partial f}{\partial x_{i}}\right\rangle$ will be the ideal of $\mathcal{E}(n)$ generated by them. Also for a germ $f$, 
$j^{k} f(x)$ will be the Taylor expansion of $f$ at the point $x$ up to degree $k$ and it is called the $k$-jet of $f$ at $x$. We will denote by $J^{k}(n, 1)$ the $\mathbb{R}$-vector space of all polynomials in $n$-coordinates up to degree $k$. In the case $k=\infty$ we understand $j^{\infty} f(x)$ the Taylor series of $f$ at $x$. Also $J^{\infty}(n, 1)$, the set of all these jets will be identified with the formal power series ring $\mathbb{R}\left[\left[x_{1}, \ldots, x_{n}\right]\right]$.

We are very grateful to the reviewer for his work and suggestions.

\section{Finite Relative Determination and Finite Determination on the Right.}

\section{Definition 1.}

(a) Let $S$ be a germ of a subset of $\mathbb{R}^{n}, f$ be a germ with $f(\overline{0})=0$ and let $k \leq \infty$. We shall say that $f$ is $k$ - determined relative to $G_{S}$ if whenever $g$ is a germ such that $j^{k} g(\overline{0})=j^{k} f(\overline{0})$ and $g-f$ vanishes at $S$, there exists $h$ in $G_{S}$ with $g=f \circ h$. In the case $S=\{\overline{0}\}$ we say that $f$ is finitely or infinitely determined on the right according to $k$ is finite or not. In general if $k$ is finite, then we say that $f$ is finitely determined relative to $G_{S}$.

(b) Let $I$ be an ideal of $\mathcal{E}(n)$ and $S=z(I)$ the germ of the common zeroes of $I$ (we suppose $\overline{0} \in S$ ). We denote by $\operatorname{rad} I$ the ideal of $\mathcal{E}(n)$ consisting of all germs vanishing at $S$, and we say that $I$ is radical if $I=\operatorname{rad} I$.

Lemma 2 (Artin-Rees). If $I$ is an ideal of $\mathbb{R}[[x]]=\mathbb{R}\left[\left[x_{1}, \ldots, x_{n}\right]\right]$, there exists $k$ such that $I \cap M^{m}=M^{m-k}\left(I \cap M^{k}\right)(\forall m \geq k)$.

We shall denote $\mathcal{A}(I)$ the minimum $k$ satisfying the equality of Lemma 2. Consider $\mathbb{R}[[x]]$ the algebra of formal power series, $M$ its maximal ideal, and the canonical projection $\pi: \mathcal{E}(n) \longrightarrow \mathbb{R}[[x]]$ which sends a germ to its Taylor infinite series and $J$ an ideal of $\mathcal{E}(n)$, we will get by Artin-Rees lemma for $l=\mathcal{A}(\pi(J)), M^{m} \cap \pi(J)=M^{m-l}\left(M^{l} \cap \pi(J)\right), \forall m \geq l$. Hence applying $\pi^{-1}$ to the above equality and intersecting each member with $J$ we get

$$
J \cap m(n)^{m}=m(n)^{m-l}\left(J \cap m(n)^{l}\right)+J \cap m(n)^{\infty} \quad(\forall m \geq l) .
$$

We shall denote $\mathcal{A}(J)$ the minimum $l$ satisfying this equality, therefore $\mathcal{A}(J) \leq \mathcal{A}(\pi(J))$.

Since $m(n)^{k} \supseteq \operatorname{ker} \pi$, then $\pi\left(J \cap m(n)^{k}\right)=\pi(J) \cap \pi\left(m(n)^{k}\right)=\pi(J) \cap M^{k}$. If we apply the epimorphism $\pi$ to the equality (*), we get $M^{m} \cap \pi(J)=$ $M^{m-l}\left(M^{l} \cap \pi(J)\right), \forall m \geq l$. Therefore $\mathcal{A}(\pi(J)) \leq \mathcal{A}(J)$ and hence $\mathcal{A}(J)=$ $\mathcal{A}(\pi(J))$.

In case $I$ is a radical ideal of $\mathcal{E}(n)$, we get in fact $I \cap m(n)^{m}=$ $m(n)^{m-k}\left(I \cap m(n)^{k}\right) \forall m \geq k$. 
Theorem 3. Consider I a finitely generated ideal of $\mathcal{E}(n)$. Then for any $k<\infty, I \cap m(n)^{k}$ is also finitely generated.

Proof. Consider $g_{1}, \ldots, g_{s}$ generators of $I$ and let $f=\sum_{i=1}^{s} h_{i} g_{i}$. Then we have

$$
\begin{aligned}
f=\sum_{i=1}^{s} h_{i}^{(k)} g_{i} & +\sum_{i=1}^{s} h_{i}^{[k]} g_{i}, \\
& \text { where } h_{i}^{(k)} \text { is the }(k-1)-\text { jet of } h_{i} \text { and } h_{i}^{[k]}=h_{i}-h_{i}^{(k)} .
\end{aligned}
$$

Hence as vector spaces $I=V+m(n)^{k} I$, where $V$ is the vector space generated by $\left\{x^{I} g_{i}\right\}$ with $|I| \leq k-1$. Therefore $I \cap m(n)^{k}=V \cap m(n)^{k}+$ $m(n)^{k} I$. It is clear that a basis of the subspace $V \cap m(n)^{k}$ of $V$ and the generators of $m(n)^{k} I$ generate $I \cap m(n)^{k}$ as an ideal of $\mathcal{E}(n)$.

Theorem 4. Suppose $I$ is a radical ideal of $\mathcal{E}(n), I \cap m(n)^{k}$ a finitely generated ideal and $I \cap m(n)^{k} \subseteq \operatorname{Im}(n)\left\langle\frac{\partial f}{\partial x_{i}}\right\rangle$ with $k \geq \mathcal{A}(I)$. Then $f$ is $k$ determined relative to $G_{S}$, where $S=z(I)$.

Theorem 5. Let $f$ be a $k$-determined germ relative to $G_{S}, S=z(I)$ and $I$ a radical finitely generated ideal. Then $I \cap m(n)^{k+1} \subseteq I\left\langle\frac{\partial f}{\partial x_{i}}\right\rangle+m(n)^{k+2} \cap I$.

Joining Theorems 4 and 5 we get for $I$ a finitely generated ideal, the following:

Theorem 6. Let $f$ be a germ, I a finitely generated radical ideal, $S=z(I)$, and $k \geq \mathcal{A}(I)$. Then $f$ is finitely determined relative to $G_{S}$ if and only if there exists a number $l$ greater or equal than $k$ such that $m(n)^{l} \cap I \subseteq I$ $\left\langle\frac{\partial f}{\partial x_{i}}\right\rangle$.

The proofs of the above theorems can be found in $[\mathbf{K}]$, Theorems 11 and 15 .

We can change Theorem 4 in the following way.

Theorem 7. Let $I$ be a radical ideal, $k=\mathcal{A}(I)$ and suppose that $I \cap$ $m(n)^{k}$ is finitely generated. Let $l$ be a natural number such that $m(n)^{l} I \subseteq$ $m(n) I\left\langle\frac{\partial f}{\partial x_{i}}\right\rangle$. Then $f$ is $(k+l-1)$ determined relative to $G_{S}$, where $S=z(I)$.

Proof. Let $g$ be a germ with $g \equiv f$ on $S$ and $j^{k+l-1} g(0)=j^{k+l-1} f(0)$.

If we define the trivial homotopy $F(x, t)=(1-t) f(x)+t g(x)$ we get $\frac{\partial F}{\partial t}=g-f \in m(n)^{k+l} \cap I$ and $\frac{\partial f}{\partial x_{i}}=\frac{\partial F}{\partial x_{i}}+t\left(\frac{\partial f}{\partial x_{i}}-\frac{\partial g}{\partial x_{i}}\right)$. 
Since $m(n)^{k+l} \cap I \subseteq m(n)^{l} I$ and $\operatorname{Im}(n)^{p-1} \subseteq I \cap m(n)^{p} \forall p$, we get

$$
\begin{aligned}
\left(m(n)^{k+l} \cap I\right) \mathcal{E}(n+1) \subseteq m(n) I\left\langle\frac{\partial F}{\partial x_{i}}\right\rangle \mathcal{E}(n+1) & \\
& +m(n)\left(m(n)^{k+l} \cap I\right) \mathcal{E}(n+1)
\end{aligned}
$$

By Nakayama's lemma we arrive to the inclusion:

$$
\left(m(n)^{k+l} \cap I\right) \mathcal{E}(n+1) \subseteq m(n) I\left\langle\frac{\partial F}{\partial x_{i}}\right\rangle \mathcal{E}(n+1) .
$$

Hence $\frac{\partial F}{\partial t}=\sum h_{i}(x, t) \frac{\partial F}{\partial x_{i}}$ with $h_{i}(x, t) \equiv 0$ for $x \in S, t$ near $t_{0}$ ( $t_{0}$ fixed). We now proceed in the usual way.

\section{Remark 1.}

(a) If $I=m(n)$ then $k=1$ and we get that $m(n)^{l+1} \subseteq m(n)^{2}\left\langle\frac{\partial f}{\partial x_{i}}\right\rangle$ implies $f$ is $l$ - determined on the right $([\mathbf{M}])$.

(b) If $I=\left\langle x_{1}, \ldots, x_{s}\right\rangle$ then $k=1$ and we get that $m(n)^{l} I \subseteq m(n) I\left\langle\frac{\partial f}{\partial x_{i}}\right\rangle$ implies $f$ is $l$ - determined relative to $G_{S}, S=\{0\} \times \mathbb{R}^{n-s}([\mathbf{P}-\mathbf{L}])$.

Corollary 8. Let $f$ be a germ, $I$ a radical ideal, $k=\mathcal{A}(I)$ and $I \cap m(n)^{k}$ be finitely generated. Suppose that $m(n)^{l} \subseteq m(n)\left\langle\frac{\partial f}{\partial x_{i}}\right\rangle$. Then $f$ is $(k+l-1)$ determined relative to $G_{S}$. Hence finite determination on the right implies finite relative determination.

Proof. Since $m(n)^{l} \subseteq m(n)\left\langle\frac{\partial f}{\partial x_{i}}\right\rangle$ then $m(n)^{l} I \subseteq m(n) I\left\langle\frac{\partial f}{\partial x_{i}}\right\rangle$. We now use Theorem 7.

We are now interested in determining for which ideals $I$ we have the converse of Corollary 8. For this purpose we need the following:

Theorem 9. Let $A$ be a commutative ring, $I, J, K$ ideals of $A$ with $I=$ $\left\langle g_{1}, \ldots, g_{k}\right\rangle$. Suppose that $a g_{i}=0$ for all $i$ and $a \in J^{k}+K$ implies $a=0$. Then if $I J \subset I K$ hence $J^{k} \subseteq K$.

Proof. Let $m_{1}, \ldots, m_{k}$ be arbitrary elements of $J$, then $g_{i} m_{i}=\sum_{j=i}^{k} g_{j} d_{i j}$ $\forall i$ with $1 \leq i \leq k \quad\left(d_{i j} \in K\right)$. In matricial notation we can write

$$
C\left(\begin{array}{c}
g_{1} \\
\vdots \\
g_{k}
\end{array}\right)=\left(\begin{array}{c}
0 \\
\vdots \\
0
\end{array}\right) \text { where } C=\left(\delta_{i j} m_{i}-d_{i j}\right) .
$$

If we multiply $(*)$ by the adjoint of $C$ we get $(\operatorname{det} C) g_{i}=0 \forall i$, but $\operatorname{det} C=m_{1} \cdots m_{k}+b$ with $b \in K$. Hence by hypothesis $\operatorname{det} C=0$ and then $m_{1} \cdots m_{k} \in K$, therefore $J^{k} \subseteq K$. 
Corollary 10. Let $A=\mathcal{E}(n), J=m(n)^{l},\left(\right.$ or $\left.J=m(n)^{\infty}\right), K=\left\langle\frac{\partial f}{\partial x_{i}}\right\rangle$ and $I$ ideal with $I=\left\langle g_{1}, \ldots, g_{k}\right\rangle$. Suppose that $h g_{i}=0$ for all $i$ and $h \in m(n)^{l k}+\left\langle\frac{\partial f}{\partial x_{i}}\right\rangle\left(\right.$ or $\left.h \in m(n)^{\infty}+\left\langle\frac{\partial f}{\partial x_{i}}\right\rangle\right)$ implies $h \equiv 0$. Then if $I m(n)^{l} \subseteq$ $I\left\langle\frac{\partial f}{\partial x_{i}}\right\rangle\left(\right.$ or $\left.I m(n)^{\infty} \subseteq I\left\langle\frac{\partial f}{\partial x_{i}}\right\rangle\right)$ hence $m(n)^{l k} \subseteq\left\langle\frac{\partial f}{\partial x_{i}}\right\rangle\left(\right.$ or $\left.m(n)^{\infty} \subseteq\left\langle\frac{\partial f}{\partial x_{i}}\right\rangle\right)$ and $f$ is $(l k+1)$-determined on the right $(\infty$ - determined on the right).

This result motivates us to find examples where $I$ is a finitely generated radical ideal satisfying the hypothesis of the above corollary.

Example 11. Let $I$ be a radical ideal generated by a non trivial analytic germ $g$. If $h g=0$ then $h \equiv 0$ and we will have finite relative determination implies determination on the right $\left(h g \equiv 0 \Longrightarrow h^{-1}(0) \cup g^{-1}(0)=\mathbb{R}^{n}\right.$ but $g^{-1}(0)$ is a closed set with empty interior, therefore $\left.h^{-1}(0)=\mathbb{R}^{n}\right)$.

Example 12. Consider in $\mathcal{E}(3)$ the ideal $I$ generated by $\left\{x_{1} x_{2}, x_{1} x_{3}, x_{2} x_{3}\right\}$. It is easy to see that $I$ is radical and $\mathcal{A}(I)=2$. Moreover if we denote $P_{1}=x_{1} x_{2}, P_{2}=x_{1} x_{3}$ and $P_{3}=x_{2} x_{3}$,we get for $i \neq j, i \neq k, j \neq k$ that the closure of $z\left(P_{i}\right) \cap z\left(P_{j}\right)-z\left(P_{k}\right)$ is a plane and does not contain $z(I)$, which is the union of the three axes, hence it does not satisfy the hypothesis of Theorem $20[\mathbf{K}]$, but the conclusion is still true. We give a proof since it is important for the converse of Corollary 8.

Proposition 13. With the above notation, if $f$ is $m$-determined relative to $G_{S}$, where $S=z(I)$ are the coordinate axes, then $f$ is $(2 m-2)$-determined on the right.

Proof. By Theorem $15([\mathbf{K}])$ we know that $m(3)^{m+1} \cap I \subseteq I\left\langle\frac{\partial f}{\partial x_{i}}\right\rangle$ which in this case is equivalent to $\operatorname{Im}(3)^{m-1} \subseteq I\left\langle\frac{\partial f}{\partial x_{i}}\right\rangle$. We shall show that $m(3)^{2 m-1} \subseteq\left\langle\frac{\partial f}{\partial x_{i}}\right\rangle m(3)^{2}$ and hence $f$ is $(2 m-2)$-determined on the right. Any mixed monomial of $m(3)^{2 m-1}$ has a factor of $I$ times a monomial of degree $2 m-3$, hence for $m \geq 2$ it is contained in the Jacobian ideal. We now give the proof for $x_{1}^{2 m-1}$, the other two are similar,

(*) $x_{1}^{m-1}\left(x_{1} x_{2}\right)=x_{1} x_{2} \sum_{j=1}^{3} \frac{\partial f}{\partial x_{j}} h_{1 j}+x_{1} x_{3} \sum_{j=1}^{3} \frac{\partial f}{\partial x_{j}} h_{2 j}+x_{2} x_{3} \sum_{j=1}^{3} \frac{\partial f}{\partial x_{j}} h_{3 j}$.

If we denote $\phi=x_{1}^{m-1}-\sum_{i=1}^{3} \frac{\partial f}{\partial x_{j}} h_{1 j}$ we get that the zeroes of $\phi$ contain $\left\{x_{3}=0\right\}$ and the zeroes of $x_{1} \phi$ contain $\left\{x_{3}=0\right\} \cup\left\{x_{1}=0\right\}$, hence $x_{1} \phi \in \hat{I}=$ $I$. From $(*)$ and the definition of $\phi, x_{1}^{m}=x_{1} \sum_{j=1}^{3} \frac{\partial f}{\partial x_{j}} h_{1 j}+x_{1} \phi$, therefore $x_{1}^{2 m-1} \in m(3)^{2}\left\langle\frac{\partial f}{\partial x_{i}}\right\rangle$ and $f$ is $(2 m-2)$-determined. 
Remark 2. By Corollary 10, since $I=\hat{I}=\left\langle x_{1} x_{2}, x_{1} x_{3}, x_{2} x_{3}\right\rangle$ then $\operatorname{Im}(3)^{m-1} \subseteq I\left\langle\frac{\partial f}{\partial x_{i}}\right\rangle$ implies $m(3)^{3(m-1)} \subseteq\left\langle\frac{\partial f}{\partial x_{i}}\right\rangle$ and $f$ is $(3 m-2)$-determined on the right.

Definition 14. Let $f_{1}, \ldots, f_{r}$ be germs in $m(n)$. We say that they are linearly independent if their gradients denoted by $\nabla f_{1}, \ldots, \nabla f_{r}$ are linearly independent at the origin.

Lemma 15. Let $f_{1}, \ldots, f_{r}$ be linearly independent germs. Then the ideal I generated by them is radical.

Proof. Let $H$ be the germ of the common zeroes of $I$ and $P_{r+1}, \ldots, P_{n}$ linear polynomials such that $\nabla f_{1}(\overline{0}), \ldots, \nabla f_{r}(\overline{0}), \nabla P_{r+1}(\overline{0}), \ldots, \nabla P_{n}(\overline{0})$ is a basis of $\mathbb{R}^{n}$. Thus $\phi=\left(f_{1}, \ldots, f_{r}, P_{r+1}, \ldots, P_{n}\right)$ is a germ of diffeomorphism. Let $f \in \widehat{I}$ hence $f \equiv 0$ on $H$ if and only if $f \circ \phi^{-1} \equiv 0$ on $\{0\} \times \mathbb{R}^{n-r}$. By Hadamard's lemma we get $f \circ \phi^{-1}\left(x_{1}, \ldots, x_{n}\right)=\sum_{i=1}^{r} x_{i} g_{i}$, therefore $f=\sum_{i=1}^{r} f_{i}\left(g_{i} \circ \phi\right)$, and hence $f$ belongs to the ideal $I$.

Lemma 16. Let $I_{1}, \ldots, I_{r}$ be radical ideals in $\mathcal{E}(n)$. Then their intersection is also a radical ideal.

Proof. In general $\operatorname{rad}\left(\cap_{i=1}^{r} I_{i}\right) \subseteq \cap_{i=1}^{r} \operatorname{rad} I_{i}$, hence we get

$$
\cap_{i=1}^{r} I_{i} \subseteq \operatorname{rad} \cap_{i=1}^{r} I_{i} \subseteq \cap_{i=1}^{r} \operatorname{rad} I_{i}=\cap_{i=1}^{r} I_{i} .
$$

Therefore the equality $\cap_{i=1}^{r} I_{i}=\operatorname{rad} \cap_{i=1}^{r} I_{i}$.

Lemmas 15 and 16 generate a special collection of algebraic sets. They are called bouquets of subspaces.

Example 17. Consider $I \subseteq \mathcal{E}(3)$ the ideal generated by $x$ and $y z$, hence $z(I)$ is the union of the $y$-axis and $z$-axis, they are not in general position (in $\mathbb{R}^{3}$ ). By Lemma $16, I$ is clearly a radical ideal since $I=I_{1} \cap I_{2}$ where $I_{1}=\langle x, y\rangle$ and $I_{2}=\langle x, z\rangle$.

Definition 18. Let $I$ be a finitely generated ideal of $\mathcal{E}(n), I=\left\langle g_{1}, \ldots, g_{k}\right\rangle$. We say that $I$ is integral if $S=z(I)$ is nowhere dense.

We now arrive at the main theorem of this section.

Theorem 19. Let $I$ be a finitely generated ideal of $\mathcal{E}(n)$ which is radical. Then if $f$ is finitely determined relative to $G_{S}, S=z(I)$, hence $f$ is finitely determined on the right.

Proof. Suppose $I=\left\langle g_{1}, \ldots, g_{k}\right\rangle$ and that $h g_{i} \equiv 0 \forall i$. Therefore $z(h) \cup$ $z\left(g_{i}\right)=\mathbb{R}^{n} \forall i$ and hence $z(h) \cup z(I)=\mathbb{R}^{n}$. Since $I$ is an integral ideal, see $[\mathbf{R}], z(h)=\mathbb{R}^{n}$ and hence $h \equiv 0$. On the other side there exists a natural number $p$ such that $m(n)^{p} I \subset\left\langle\frac{\partial f}{\partial x_{i}}\right\rangle I$. By Corollary 10, we get $m(n)^{p k} \subset\left\langle\frac{\partial f}{\partial x_{i}}\right\rangle$ and therefore $f$ is $(p k+1)$-determined on the right. 
Corollary 20. Consider $I_{1}, \ldots, I_{r}$ ideals each of them generated by linearly independent linear polynomials and $S$ the union of their common zeroes (bouquet of subspaces). Then a germ $f$ is finitely determined on the right if and only if $f$ is finitely determined relative to $G_{S}$.

We finish this section with an observation about homogeneous polynomials.

Proposition 21. Let $h_{1}, \ldots, h_{k}$ be homogeneous polynomials of degree $s_{1}, \ldots, s_{k}$ respectively and let $s$ be the maximum of these degrees. Hence if $I$ is the ideal generated by $h_{1}, \ldots, h_{k}$ we get $\mathcal{A}(I) \leq s$.

Proof. We have to show that $\left(I \cap m(n)^{s}\right) m(n)^{r}=I \cap m(n)^{s+r} \forall r \geq 0$. Let $f \in I \cap m(n)^{s+r}$, hence we have the following equalities.

$$
f=h_{1} g_{1}+\ldots+h_{k} g_{k}
$$

$$
0=j^{s+r-1} f(0)=h_{1} j^{r+s-1-s_{1}} g_{1}(0)+\cdots+h_{k} j^{r+s-1-s_{k}} g_{k}(0) .
$$

Sustracting $(* *)$ from $(*)$ we get $f=h_{1} \quad \tilde{g_{1}}+\cdots+h_{k} \tilde{g_{k}}$, where $\tilde{g_{i}} \in$ $m(n)^{r+s-s_{i}}$.

Hence each $\tilde{g}_{i}$ is a sum of elements of the form $\tilde{h_{i}^{j}} \underset{h_{i}^{j}}{\tilde{h^{j}}}$, with $\tilde{h}_{i}^{j} \in m(n)^{r}$ $\approx$

and $h_{i}^{j}$ is a homogeneous monomial of degree $s-s_{i}$.

Therefore $f$ is a sum of elements of the form $\left(\underset{h_{i}}{\widetilde{h_{i}^{j}}}\right) \widetilde{h_{i}^{j}}$, with $\left(\underset{h_{i}}{\approx_{i}^{j}}\right) \in$ $I \cap m(n)^{s}$, so $f \in\left(I \cap m(n)^{s}\right) m(n)^{r}$. We have shown that $I \cap m(n)^{s+r} \subseteq$ $\left(I \cap m(n)^{s}\right) m(n)^{r} \forall r \geq 0$. The other inclusion is obvious.

\section{Infinite determinacy on germs of closed subsets of $\mathbb{R}^{n}$.}

In this setction we will assume that $S$ is a germ of a closed subset of $\mathbb{R}^{n}$ such that the origin is an accumulation point of $S$.

Definition 22. Let $S \subseteq \mathbb{R}^{n}$ be a germ of a closed set such that $\overline{0}$ is an accumulation point of $S$. We say that a germ $f$ in $\mathcal{E}(n)$ is $S$-infinitely determined if given a germ $g$ such that $j^{\infty} g(x)=j^{\infty} f(x) \forall x \in S$ there exists a germ of a diffeomorphism $\phi$ such that $g=f \circ \phi$.

We denote by $\mathcal{E}(S, n)$ the ideal of $\mathcal{E}(n)$ consisting of the germs $f$ such that $j^{\infty} f(x)=0$ for all $x \in S$. If $f$ is a germ in this ideal, we can write $f=g h$ where $\{g, h\} \subseteq m(n)^{\infty}$ and $h(x)>0$ for $x \neq 0$. Then $j^{\infty} g(x)=0 \forall$ $x \neq 0, x \in S$ and therefore $\mathcal{E}(S, n) \subseteq \mathcal{E}(S, n) m(n)^{\infty}$. We get in fact the equality.

Remark 3. If $f \in \mathcal{E}(S, n)$ then for all multi-index $I, \frac{\partial^{|I|} f}{\partial x^{I}} \in \mathcal{E}(S, n)$. 
Definition 23. A germ $f$ is $S$-infinitesimally stable if $\mathcal{E}(S, n) \subseteq\left\langle\frac{\partial f}{\partial x_{i}}\right\rangle \mathcal{E}(S, n)$.

Theorem 24. Let $S$ be a germ of a closed subset of $\mathbb{R}^{n}$ such that the origin is an accumulation point of $S$. If $f$ is $S$-infinitesimally stable then $f$ is $S$-infinitely determined.

Proof. Let $g(x)$ be a germ such that $j^{\infty} g(x)=j^{\infty} f(x) \forall x \in S$. We define the homotopy $F(x, t)=\operatorname{tg}(x)+(1-t) f(x)$. Consider the following $\mathcal{E}(n+1)-$ modules $N=\mathcal{E}(n+1)\left\langle\frac{\partial f}{\partial x_{i}}\right\rangle$ and $K=\mathcal{E}(n+1)\left\langle\frac{\partial F}{\partial x_{i}}\right\rangle$. If $h \in N$, we have $h(x, t)=\sum_{i=1}^{n} \frac{\partial f}{\partial x_{i}}(x) h_{i}(x, t)=\sum_{i=1}^{n} \frac{\partial F}{\partial x_{i}}(x, t) h_{i}(x, t)+$ $t \sum_{i=1}^{n} \frac{\partial(f-g)}{\partial x_{i}}(x) h_{i}(x, t)$. Since $\frac{\partial(f-g)}{\partial x_{i}}(x) \in \mathcal{E}(S, n) \subseteq\left\langle\frac{\partial f}{\partial x_{i}}\right\rangle \mathcal{E}(S, n)$, we get $N \subseteq K+\mathcal{E}(S, n) N$, and by Nakayama's lemma, $N \subseteq K$ and hence $\mathcal{E}(S \times \mathbb{R}$, $n+1)\left\langle\frac{\partial f}{\partial x_{i}}\right\rangle \mathcal{E}(n+1) \subseteq \mathcal{E}(S \times \mathbb{R}, n+1)\left\langle\frac{\partial F}{\partial x_{i}}\right\rangle \mathcal{E}(n+1)$.

Since $g-f \in \mathcal{E}(S \times \mathbb{R}, n+1) \quad\left\langle\frac{\partial f}{\partial x_{i}}\right\rangle \mathcal{E}(n+1)$, hence $\frac{\partial F}{\partial t} \in \mathcal{E}(S \times \mathbb{R}, n+1)\left\langle\frac{\partial F}{\partial x_{i}}\right\rangle \mathcal{E}(n+1)$. We now proceed in the usual way.

Proposition 25. If $f$ is a germ, finitely (infinitely) determined on the right, it is $S$-infinitesimally stable and therefore $S$-infinitely determined.

Proof. By our hypothesis we have

$$
m(n)^{\infty} \subseteq\left\langle\frac{\partial f}{\partial x_{i}}\right\rangle \text { and } \mathcal{E}(S, n) \subseteq \mathcal{E}(S, n) m(n)^{\infty} \subseteq \mathcal{E}(S, n)\left\langle\frac{\partial f}{\partial x_{i}}\right\rangle .
$$

\section{Example 26.}

(a) Let $f$ be a germ and $k$ a natural number, denote by $I_{k}$ the ideal generated by $f^{k}$. Suppose $\mathcal{E}(S, n) \subseteq I_{k}, f^{k} \in\left\langle\frac{\partial f}{\partial x_{i}}\right\rangle$ and $j^{\infty} f(x) \neq$ $0 \forall x \in T$, where the closure of $T$ is $S$. If $h \in \mathcal{E}(S, n), h=f^{k} g$ where $g \in \mathcal{E}(S, n)$. Therefore $\mathcal{E}(S, n) \subseteq \mathcal{E}(S, n)\left\langle f^{k}\right\rangle \subseteq \mathcal{E}(S, n)\left\langle\frac{\partial f}{\partial x_{i}}\right\rangle$, and thus $f$ is $S$-infinitely determined.

(b) In particular let $S=\left\{\left(x_{1}, \ldots, x_{n}\right) \mid x_{1} \leq 0\right\}$. Then for the germs $f_{1}\left(x_{1}, \ldots, x_{n}\right)=\sum_{i=1}^{n} x_{i}^{2}$, and $f_{2}\left(x_{1}, \ldots, x_{n}\right)=x_{1}$, we get $j^{\infty} f_{1}(x) \neq$ 0 and $j^{\infty} f_{2}(x) \neq 0 \forall x \in \operatorname{int} S$. Since $S=\operatorname{cl}(\operatorname{int} S)$ and $\mathcal{E}(S, n) \subseteq\left\langle f_{i}^{k}\right\rangle$ for $i=1,2$ (Proposition 5.4 of Chapter $\mathrm{V},[\mathbf{T}]$ ), and clearly $f_{i}^{k} \in\left\langle\frac{\partial f_{i}}{\partial x_{j}}\right\rangle$ for $i=1,2$, we get that $f_{1}^{k}, f_{2}^{k}$ are $S$-infinitely determined. ([P-L]).

\section{Definition 27.}

(a) Let $S$ be a closed subset of $\mathbb{R}^{n}$ (containing the origin) and $f$ a germ with $f(\overline{0})=0$. We say that $f$ satisfies a Lojasiewicz inequality for $S$ if for any $K$, a germ of a compact set with $\overline{0} \in K$, there exist constants $c>0$ and $\alpha \geq 0$ such that $|f(x)| \geq c d(x, S)^{\alpha}$ for all $x \in K$. 
(b) Let $I$ be a finitely generated ideal of $\mathcal{E}(n)$ and $S$ the germ of its common zeroes. We say that $I$ is a Lojasiewicz ideal if there exists $f$ in $I$ satisfying a Lojasiewicz inequality for $S$.

(c) Let $f \in m(n)$ and $S$ a closed subset of $\mathbb{R}^{n}$, we say that $f$ satisfies a Jacobi-Lojasiewicz condition for $S$ if $|\nabla f|$ satisfies a Lojasiewicz inequality for $S$.

Remark 4. If $\left\{f_{1}, \ldots, f_{s}\right\}$ is a set of generators of a Lojasiewicz ideal $I$, then $\sum_{i=1}^{s} f_{i}^{2}, \sum_{i=1}^{s}\left|f_{i}\right|$ and $\max \left\{f_{1}^{2}, \ldots, f_{s}^{2}\right\}$ also satisfy a Lojasiewicz inequality for $S$.

Definition 28. Let $\left(b_{i}\right)$ be a sequence of positive real numbers converging to zero. We say that a sequence of real numbers $\left(a_{i}\right)$ is flat along $\left(b_{i}\right)$ if given $r>0$ there exists a natural number $N=N(r)$ such that $\left|a_{i}\right| \leq b_{i}^{r}$ for $i \geq N$. Sequences of vectors, matrices, jets are flat along a sequence $\left(b_{i}\right)$ if each entry is flat along $\left(b_{i}\right)$. A sequence is flat along a sequence $\left(x_{i}\right)$ of nonzero vectors in $\mathbb{R}^{n}$ converging to the vector 0 if it is flat along the sequence $\left(\left|x_{i}\right|\right)$. In the case of $\infty$-jets, we ask for a uniform $N=N(r)$ for all entries. Here we are identifying $\sum_{\alpha} a_{\alpha} \frac{\left(x-x_{0}\right)^{\alpha}}{\alpha !}$ with $\left(a_{\alpha}\right)$.

Remark 5. We can change $r>0$ for $r=n, n$ a natural number since for $n>r$, we get $b_{i}^{n} \leq b_{i}^{r}\left(0 \leq b_{i} \leq 1\right)$.

We state an interesting equivalence.

Lemma 29. A germ $g$ does not satisfy a Lojasiewicz inequality for a closed subset $S$ if and only if there exists a sequence of vectors $x_{i} \in \mathbb{R}^{n}-S$ converging to the vector $\overline{0}$ such that $\left(g\left(x_{i}\right)\right)$ is flat along $\left(d\left(x_{i}, S\right)\right)$.

Remark 6. For a germ $g$ not identically zero we can choose $g\left(x_{i}\right) \neq 0 \forall i$.

Definition 30. Let $S$ be a closed subset of $\mathbb{R}^{n}$. Then $M(S, n)$ is the set of maps $\phi: \mathbb{R}^{n}-S \longrightarrow \mathbb{R}$ such that if $K$ is a germ of a compact set and $I$ is a multi-index of natural numbers, there exist constants $c>0$ and $\alpha>0$ such that $\left|\frac{\partial^{|I|} \phi}{\partial x^{I}}(x)\right| \leq c d(x, S)^{-\alpha}$ for all $x \in K-S$.

We state the following proposition (Chapter IV, Proposition 4.2 of $[\mathbf{T}]$ ).

Proposition 31. Let $\phi \in M(S, n)$ and $f \in \mathcal{E}(S, n)$. Then we can extend $\phi f$ in a unique way to a germ in $\mathcal{E}(S, n)$, denoted also by $\phi f$.

Theorem 32. Let $f$ be a germ, $S$ a germ of a closed subset of $\mathbb{R}^{n}$ such that 0 is an accumulation point of $S$. Suppose that $f$ satisfies a Jacobi-Lojasiewicz condition for $S$. Then $f$ is $S$-infinitesimally stable and therefore $S$-infinitely determined.

Proof. Consider $g=|\nabla f|^{2}$, we shall show that $\mathcal{E}(S, n) \subseteq\left\langle\frac{\partial f}{\partial x_{i}}\right\rangle \mathcal{E}(S, n)$. Let $K$ be a germ of a compact subset and $g_{1}$ be a representative of $g$; for 
each $I$ multi-index there exists $C_{I}$ constant such that $\left|\frac{\partial^{|I|}\left(\frac{1}{g_{1}}\right)}{\partial x^{I}}\right| \leq \frac{C_{I}}{\left|g_{1}(x)\right|^{(I I \mid+1)}}$ $\forall x \in K$. Since $g_{1}$ satisfies a Lojasiewicz inequality for $S$, there exist $c>0$ and $\alpha \geq 0$ such that $\left|g_{1}(x)\right| \geq c d(x, S)^{\alpha} \forall x \in K-S$ and therefore $\left|\frac{\partial^{|I|}\left(\frac{1}{g_{1}}\right)}{\partial x^{I}}\right| \leq$ $\frac{C_{I}}{c^{I I+1} d(x, S)^{\alpha(|I|+1)}} \forall x \in K-S$, hence $\frac{1}{g_{1}} \in M(S, n)$. Now for $h \in \mathcal{E}(S, n)$ and $x \notin S$ we have $h(x)=\frac{h(x)}{g_{1}(x)} g_{1}(x)$, extend $\frac{h(x)}{g_{1}(x)}$ to a germ $H$ in $\mathcal{E}(S, n)$ and $h=H g_{1}$ in $\mathcal{E}(S, n)\left\langle\frac{\partial f}{\partial x_{i}}\right\rangle$. Therefore we get $\mathcal{E}(S, n) \subseteq \mathcal{E}(S, n)\left\langle\frac{\partial f}{\partial x_{i}}\right\rangle$ and $f$ is $S$-infinitesimally stable.

Lemma 33 ([W, Lemma 3.3]). Suppose there exist a sequence $\left(w_{i}\right)$ in $J^{k}(n, 1), k \leq \infty$, a sequence $\left(x_{i}\right)$ in $\mathbb{R}^{n}-\{\overline{0}\}$ converging to the origin and a germ $f$ such that $q_{i}=w_{i}-j^{k} f\left(x_{i}\right)$ is flat along $\left(x_{i}\right)$. Then there exists a germ $g$ such that $j^{k} g\left(x_{i_{j}}\right)=w_{i_{j}}$ holds for $\left(x_{i_{j}}\right)$ subsequence of $\left(x_{i}\right)$, and $j^{\infty} g(\overline{0})=j^{\infty} f(\overline{0})$.

Lemma 34. Suppose there exist a sequence $\left(w_{i}\right)$ in $J^{k}(n, 1), k \leq \infty, a$ sequence $\left(x_{i}\right)$ in $\mathbb{R}^{n}-S$ converging to zero and a germ $f \in \mathcal{E}(n)$ such that $\left(q_{i}\right)=\left(w_{i}-j^{k} f\left(x_{i}\right)\right)$ is flat along $\left(d\left(x_{i}, S\right)\right)$, where $S$ is a closed subset of $\mathbb{R}^{n}(\overline{0} \in S)$. Then there exists a germ $g \in \mathcal{E}(n)$, such that $j^{\infty} g(x)=$ $j^{\infty} f(x) \forall x \in S$ and $j^{k} g\left(x_{i}\right)=w_{i}$ holds for a subsequence of $\left(x_{i}\right)$.

Proof. If $k$ is finite, then we transform $q_{i}$ into an $\infty$-jet in such a way that all the terms of order greater than $k$ of $q_{i}$ are zero. Thus we will assume $k=\infty$.

We define $Q$, a Taylor field, by $q_{i}$ at $x_{i}$ and by the zero series on $S$. We want to show that $Q$ is a $C^{\infty}$ Whitney field. It is enough to show (Proposition 1.5 of Chapter IV, $[\mathbf{T}]$ ) for each $m$ and each multi-index $I$ with $|I| \leq m$, that $\left(R_{y}^{m} Q\right)^{I}(x)=o\left(|x-y|^{m-|I|}\right)$, where $\left(R_{y}^{m} Q\right)^{I}(x)=$ $Q^{I}(x)-\sum_{|L| \leq m-|I|} Q^{I+L}(y) \frac{(x-y)^{L}}{L !}$.

If $\{x, y\} \subseteq S$ then the proof is obvious. In the case $\{x, y\} \subseteq\left\{x_{i}\right\} \cup\{\overline{0}\}$ we proceed as in the proof of Lemma 3.3 of $[\mathbf{W}]$. If $\{x, y\}=\left\{x_{j}, s\right\}, s \in S$, we use the flatness of $\left(q_{i}\right)$ along $\left(d\left(x_{i}, S\right)\right)$ to obtain for each natural number $l$ another $N(l)$ such that $\left|\left(R_{s}^{m}\right)^{I}\left(x_{j}\right)\right|=\left|q_{j}^{I}\right| \leq d\left(x_{j}, S\right)^{l} \leq d\left(x_{j}, s\right)^{l}$ and $\left|\left(R_{x_{j}}^{m}\right)^{I}(s)\right| \leq\left|\sum_{|L| \leq m-|I|} q_{j}^{(I+L)} \frac{\left(s-x_{j}\right)^{L}}{L !}\right| \leq C d\left(x_{j}, s\right)^{l}$ for $j \geq N(l)$, where $C$ is a positive real number depending only on $m$ and $I$. Let $l=m+1$.

Hence, using Whitney Extension Theorem (Theorem 3.1 of Chapter IV, $[\mathbf{T}])$, there exists a smooth germ $q$ such that $j^{\infty} q(x)=0 \forall x \in S$ and $j^{\infty} q\left(x_{i}\right)=q_{i}$. If $g=f+q$, we see that $g$ has the desired properties.

Theorem 35. Let $f$ be a germ, $S$ a closed subset of $\mathbb{R}^{n}$ and $\overline{0}$ an accumulation point of $S$. Hence if $f$ is $S$-infinitely determined, then $f$ satisfies a Jacobi-Lojasiewicz condition for $S$. 
Proof. We shall prove the theorem by contradiction. Then there is a sequence $\left(x_{j}\right)$ in $\mathbb{R}^{n}-S$ converging to the origin such that $\left(\left|\nabla f\left(x_{j}\right)\right|\right)$ is flat along $\left(d\left(x_{j}, S\right)\right)$. Choose $\left(y_{j}\right)$ a sequence of regular values of $f$ converging to zero and such that $\left(f\left(x_{j}\right)-y_{j}\right)$ is flat along $\left(d\left(x_{j}, S\right)\right)$. It clearly follows that $\left(y_{j}, 0\right)-\left(f\left(x_{j}\right), \nabla f\left(x_{j}\right)\right)$ is flat along $\left(d\left(x_{j}, S\right)\right)$.

If we denote $q_{j}=\left(y_{j}, 0\right)-\left(f\left(x_{j}\right), \nabla f\left(x_{j}\right)\right)$ and setting $k=1$ in the previous lemma, there exists a germ $g$ such that $j^{1} g\left(x_{j}\right)=\left(y_{j}, 0\right)$ and $g-f \in$ $\mathcal{E}(S, n)$. Now since $f$ is $S$-infinitely determined, $f$ and $g$ must have the same critical and regular values, which is not the case, since the points $y_{j}$ are regular values for $f$ but critical values for $g$.

As a consequence of Theorems 24, 32 and 35 we get the main theorem of part II:

Theorem 36. Let $f \in \mathcal{E}(n)$. The concepts of $S$-infinitesimally stability, $S$ infinite determinacy and the Jacobi-Lojasiewicz condition at $S$ are equivalent for the germ $f$ and $S$ a germ of a closed subset of $\mathbb{R}^{n}$ with $\overline{0}$ an accumulation point of $S$.

\section{A special case.}

Definition 37. Let $S$ be a germ of a closed subset of $\mathbb{R}^{n}$ such that $\overline{0} \in$ $c l$ (int). We say that a germ $f$ is $S$-stable, if given a germ $g$ such that $g(x)=f(x) \forall x \in S$, there exists a germ of a diffeomorphism $\phi \in G_{S}$ such that $g=f \circ \phi$.

Note that if $c l$ (int) $=S$, the previous definition is apparently much stronger than Definition 23. In this case $f(x)=g(x) \forall x \in S$ and $j^{\infty} g(x)=$ $j^{\infty} f(x) \forall x \in S$ are equivalent but now we restrict ourselves to the group $G_{S}$, hence the diffeomorphism must be the identity on $S$.

\section{Example 38.}

(a) Let $S=\left\{(x, y) \in \mathbb{R}^{2} \mid x \leq 0\right.$ and $\left.y=0\right\}$, then $S$ is closed but $\overline{0} \notin$ $c l(\operatorname{int} S)$.

(b) Let $S=\left\{(x, y) \in \mathbb{R}^{2} \mid x^{4}-x^{3}-x y^{2} \geq 0\right\}$, in this case $S=c l($ int $S)$.

(c) Let $S=\left\{(x, y, z) \in \mathbb{R}^{3} \mid z\left(x^{2}+y^{2}\right)-x^{3} \leq 0\right\}$, in this case $\overline{0} \in \operatorname{cl}(\operatorname{int} S)$ but clearly $\operatorname{cl}($ int $S) \neq S$.

For $S$ any germ of subset of $\mathbb{R}^{n}$ containing the origin, we let $C_{S}\left(\mathbb{R}^{n}\right)$ be the $\mathbb{R}$-algebra of germs constant at $S$. It is a local algebra with maximal ideal $m(S)$ consisting of germs of $C_{S}\left(\mathbb{R}^{n}\right)$ vanishing at $S$. In fact $m(S)$ is an ideal of $\mathcal{E}(n)$.

Remark 7. If $f \in m(S)$ and $S=\operatorname{cl}($ int $S)$ we have $f \in m(n)^{\infty}$ and $\frac{\partial^{|I|} f}{\partial x^{I}} \in$ $m(S)$ for all multi-index $I$. We also get in this case the equality $m(S)=$ $m(n)^{\infty} m(S)$. 
Lemma 39. Let $S$ a subset of $\mathbb{R}^{n}$. Suppose $S_{0}$ is a nonempty open subset of $S$. Then $\operatorname{cl}\left(S_{0}\right)=\operatorname{cl}(\operatorname{int} S)$ if and only if int $\left(S-S_{0}\right) \subseteq \operatorname{cl}\left(S_{0}\right)$.

Proof. We decompose int $S$ in the following way: $\operatorname{int} S=S_{0} \cup \operatorname{int}\left(S-S_{0}\right) \cup T$, where $\operatorname{int} T=\emptyset$. Then $c l(\operatorname{int} S)=\operatorname{cl}\left(S_{0}\right) \cup \operatorname{cl}\left(\operatorname{int}\left(S-S_{0}\right)\right)$, since $c l(T) \subseteq$ $c l\left(S_{0} \cup \operatorname{int}\left(S-S_{0}\right)\right)$. Hence $c l(\operatorname{int} S)=c l\left(S_{0}\right)$ if and only if $c l\left(\operatorname{int}\left(S-S_{0}\right)\right) \subseteq$ $\operatorname{cl}\left(S_{0}\right)$ and this is equivalent to $\operatorname{int}\left(S-S_{0}\right) \subseteq \operatorname{cl}\left(S_{0}\right)$.

Definition 40. Let $A$ be a closed subset of $\mathbb{R}^{n}$. We say that $A$ is good if there exists a locally finite partition $\mathcal{P}$ of $A$ into $C^{0}$-submanifolds of $\mathbb{R}^{n}$, called strata, such that if $\mathcal{X} \in \mathcal{P}$ and $\operatorname{dim} \mathcal{X}<n$, then there exists a non void open stratum $\mathcal{Y} \in \mathcal{P}$ such that $\mathcal{X} \subset \operatorname{cl}(\mathcal{Y})$.

We clearly have the next:

Proposition 41. Suppose that $S$ is a good subset of $\mathbb{R}^{n}$. Then $\operatorname{cl}(S)=$ $c l($ int $S)$.

Joining Lemma 39 and Proposition 41 we get the following:

Proposition 42. Let $P_{1}, \ldots, P_{s}$ be real continous functions on $\mathbb{R}^{n}$ such that $S=\left\{x \mid P_{i}(x) \leq 0 \forall i\right\}$ is good and define $S_{0}=\left\{x \mid P_{i}(x)<0 \forall i\right\}$. Suppose $\operatorname{int}\left(S-S_{0}\right) \subseteq \operatorname{cl}\left(S_{0}\right)$. Then $\operatorname{cl}\left(S_{0}\right)=S$.

Remark 8. If $P_{1}, \ldots, P_{s}$ are real analytic functions on $\mathbb{R}^{n}$ then $S=$ $\left\{x \mid P_{i}(x) \leq 0 \forall i\right\}$ will be good if we have for a decomposition of $S$, that whenever $T$ is a stratum of lower dimension, then there exists a nonempty open stratum $T^{\prime}$ such that $T \subset \operatorname{cl}\left(T^{\prime}\right)$. Obviously there are more examples of good sets than the semianalytical ones. For this purpose see for instance Sections 1 and 2 of $[\mathbf{V}-\mathbf{M}]$.

We remind here the following:

Definition 43. Suppose that $S$ is a closed subset of $\mathbb{R}^{n}$ containing the origin and such that $S=c l(\operatorname{int} S)$. We say that $f$ is $S$-infinitesimally stable if $m(S) \subseteq\left\langle\frac{\partial f}{\partial x_{i}}\right\rangle m(S)$.

Theorem 44. Suppose $S$ is a closed subset of $\mathbb{R}^{n}$ such that $\overline{0} \in S$ and $S=c l(\operatorname{int} S)$. If $f$ is $S$-infinitesimally stable then $f$ is $S$-stable.

Proof. Following the proof of Theorem 24 we start with $g$ a germ such that $g(x)=f(x) \forall x \in S$, therefore $\frac{\partial^{|I|} f}{\partial x^{I}}(x)=\frac{\partial^{|I|} g}{\partial x^{I}}(x) \forall x \in S$, and we arrive to the inclusion $m(S \times \mathbb{R})\left\langle\frac{\partial f}{\partial x_{i}}\right\rangle C_{S \times \mathbb{R}}\left(\mathbb{R}^{n} \times \mathbb{R}\right) \subseteq m(S \times \mathbb{R})\left\langle\frac{\partial F}{\partial x_{i}}\right\rangle C_{S \times \mathbb{R}}\left(\mathbb{R}^{n} \times \mathbb{R}\right)$.

Since $\frac{\partial F}{\partial t}=g-f \in m(S \times \mathbb{R}) \subseteq m(S \times \mathbb{R})\left\langle\frac{\partial f}{\partial x_{i}}\right\rangle C_{S \times \mathbb{R}}\left(\mathbb{R}^{n} \times \mathbb{R}\right)$, then $\frac{\partial F}{\partial t}(x, t)=\sum_{i=1}^{n} h_{i}(x, t) \frac{\partial F}{\partial x_{i}}(x, t)$, with $h_{i}(x, t) \in m(S \times \mathbb{R})$, hence $h_{i}(x, t)=0$ $\forall(x, t) \in S \times \mathbb{R}$. When we integrate, the required diffeomorphism will belong to $G_{S}$. 
Proposition 45. If $f \in \mathcal{E}(n)$ is a finitely (infinitely) determined on the right, then $f$ is $S$-infinitesimally stable and therefore $S$-stable for $S=$ $c l($ int $S)$.

Proof. Since $m(S)=m(n)^{\infty} m(S)$ and $m(n)^{k} \subseteq\left\langle\frac{\partial f}{\partial x_{i}}\right\rangle$ for some $k \leq \infty$, we get that $m(S) \subseteq\left\langle\frac{\partial f}{\partial x_{i}}\right\rangle m(S)$. We now use Theorem 44 .

Definition 46. Let $P$ be a polynomial in variables $x_{1}, \ldots, x_{n}$. We say that $P$ is quasihomogeneous of degree $l$ and weights $k_{1}, \ldots, k_{n}$ if $P\left(t^{k_{1}} x_{1}, \ldots, t^{k_{n}} x_{n}\right)=t^{l} P\left(x_{1}, \ldots, x_{n}\right)$.

For $P$ quasihomogeneous we get $\frac{\partial P}{\partial x_{j}}\left(t^{k_{1}} x_{1}, \ldots, t^{k_{n}} x_{n}\right)=$ $t^{l-k j} \frac{\partial P}{\partial x_{j}}\left(x_{1}, \ldots, x_{n}\right)$.

Also if we write $P=\sum a_{I} x^{I}$, for a quasihomogeneous polynomial we obtain for any multi-index $I=\left(i_{1}, \ldots, i_{n}\right), i_{1} k_{1}+\ldots+i_{n} k_{n}=l\left(a_{I} \neq 0\right)$.

Theorem 47. Let $P(x)$ be a quasihomogeneous polynomial and $S$ a closed subset of $\mathbb{R}^{n}$ containing the origin and such that $S=c l(\operatorname{int} S)$. Suppose that $m(S) \subseteq\langle P\rangle$ and that $z(P) \cap \operatorname{int} S=\phi$. Then $P$ is $S$-infinitesimally stable. In the case $S=\{x \mid P(x) \leq 0\}$ is a good semialgebraic set, we can skip the equality $z(P) \cap \operatorname{int} S=\phi$.

Proof. By hypothesis we get $m(S) \subseteq\langle P\rangle$ and $P \in\left\langle\frac{\partial P}{\partial x_{i}}\right\rangle$, this together with $z(P) \cap$ int $S=\phi$ give the result using Example 26. For the second part it is obvious that $z(P) \cap \operatorname{int} S=\phi$ since $S$ is a good semialgebraic set.

As in the previous section, we get the following:

Theorem 48. Let $f \in \mathcal{E}(n), S$ be a closed subset of $\mathbb{R}^{n}$ such that the origin is an accumulation point of $S$ and $S=c l(\operatorname{int} S)$. Then the concepts for $f$ of $S$-infinitesimally stability, $S$-stability and the Jacobi-Lojasiewicz condition for $S$ are equivalent.

Proof. Our Theorem 44 shows that $S$-infinitesimally stability implies $S$ stability. Now as in Theorem 32, we show that the Jacobi-Lojasiewicz condition at $S$ implies $S$-infinitesimally stability. Since Lemma 34 is true for any closed subset of $\mathbb{R}^{n}$, the proof of Theorem 35 will be true in the case $S=c l(\operatorname{int} S)$, and hence $S$-stability implies the Jacobi-Lojasiewicz condition of $f$ for $S$.

\section{References}

[K] L. Kushner, Finite determination on algebraic sets, Trans. of the Amer. Math. Soc., 331(2) (1992), 553-561. 
[M] J. Mather, Stability of $C^{\infty}$ mappings: III, finitely determined map-germs, Pub. Math. I.H.E.S., 35 (1968), 127-156.

[P] P. Porto, On relative stability of function germs, Bol. Soc. Bras. Mat., 14(2) (1983), 99-108.

[P-L] P. Porto and G. Loibel, Relative finite determinacy and relative stability of function germs, Bol. Soc. Bras. Mat., 9(2) (1978), 1-17.

[R] B. Roth, Finitely generated ideals of differentiable functions, Trans. Amer. Math. Soc., 150 (1970), 671-684.

[T] J.C. Tougeron, Ideaux de Fonctions Differentiables, Ergebnisse, Band 71 SpringerVerlag, New York, 1972.

[V-M] L. Van den Dries and C. Miller, Geometric categories and o-minimal structures, Duke Math. J., 84(2) (1996), 497-539.

[W] L. Wilson, Infinitely determined mapgerms, Can. J. Math., XXXIII(3) (1981), 671-684.

Received June 16, 1998 and revised October 19, 1998.

Facultad de Ciencias, UnAM

MÉXICO, D.F. 04510

MÉXICO

E-mail address: kushner@servidor.unam.mx

Universidade Federal de SÃo Carlos

13560-905 Sao Carlos, S.P.

BRASIL

E-mail address: bterra@power.ufscar.br 